\section{Estudo \\ CoDebate}

em Testão

Dlanejamento
Revista Estudo \& Debate, Lajeado, v. 28, n. 1, 2021. ISSN 1983-036X

DOI: http://dx.doi.org/10.22410/issn.1983-036X.v28i1a2021.2591

\title{
NÍVEL DE EDUCAÇÃO E TAXA DE NATALIDADE: UMA ANÁLISE ESPACIAL NOS MUNICÍPIOS DA REGIÃO SUL
}

\author{
Amarildo de Paula Junior ${ }^{1}$, José Rodrigo Gobi ${ }^{2}$, Rodrigo Monteiro Silva ${ }^{3}$, \\ Ilson Juliano Barreto ${ }^{4}$
}

\begin{abstract}
Resumo: $\mathrm{O}$ presente estudo tem como objetivo analisar a correlação espacial entre o nível de educação e a taxa de natalidade nos municípios da Regiáo Sul. Para isto foram utilizados dados da FIRJAN e do DATASUS para o ano de 2016, além de aplicada a metodologia de Análise Exploratória de Dados Espaciais. A escolha deste ano se deve por ser o mais recente até entáo com variáveis que permitem aplicar a metodologia em níveis municipais. $\mathrm{Na}$ análise global, verificou-se uma correlação inversa entre o Índice FIRJAN de Desenvolvimento Municipal em Educação e o número de nascimentos a cada mil habitantes. Porém em uma análise local, os principais resultados indicam a existência de um cluster "alto-baixo" no Sul e no Leste do Paraná. Enquanto, no Sul do Rio Grande do Sul há existência de um cluster "baixo-baixo".
\end{abstract}

Palavras-chave: Educação; Natalidade; Regiāo Sul.

Código JEL: I20; R10.

\section{EDUCATION LEVEL AND BIRTH RATE: A SPATIAL ANALYSIS IN THE MUNICIPALITIES OF THE SOUTHERN REGION}

\begin{abstract}
The present study seeks to analyze the spatial correlation between the level of education and birth rates in the municipalities of the South Region. For this, data were collected from FIRJAN and DATASUS for the year 2016, in addition to the methodology of Exploratory Analysis of Spatial Data. This year choice is due to being the most recent one so far with variables that allow the methodology to be applied at municipal levels. In the global analysis, there was an inverse correlation between the FIRJAN Municipal Development Index in Education and the number of births. However, in a local analysis, the main results indicate the existence of a "high-low" cluster in the South and East of Paraná. Meanwhile, in the South of Rio Grande do Sul there is a "low-low" cluster.
\end{abstract}

Keywords: Education; Birth; South Region.

JEL Code: I20; R10.

1 Mestre em Economia pela Universidade Estadual de Maringá.

2 Mestre em Economia pela Universidade Estadual de Maringá.

3 Mestre em Economia pela Universidade Estadual de Maringá.

4 Doutor em Geografia pela Universidade Estadual Paulista. 


\section{INTRODUÇÃO}

A taxa de natalidade é um tema presente em diversos estudos ao longo dos últimos séculos, uma vez que, existiram revoluçóes no âmbito produtivo e alocativo da economia mundial em decorrência das transformaçóes ocorridas ao longo dos últimos quatro séculos, profundas mudanças na dinâmica de crescimento demográfico foram observadas, sendo causadas pelo expressivo aumento da natalidade nesse período. No campo da ciência econômica, autores já no século XVIII, como Thomas Malthus, elaboraram suas contribuiçóes sobre o tema tentando entender o processo pelo qual ocorre o aumento da natalidade, ou seja, seus determinantes, bem como quais são as suas consequências sociais e econômicas.

Becker (1960) e Marshall (1985) expandiram a análise malthusiana e consideraram outros fatores pelos quais a natalidade em uma regiáo poderia se elevar. Deste modo, o debate sobre o tema ganhou novos horizontes ao evidenciar não somente a causa do processo de mudanças da taxa de natalidade em um território, mas também suas consequências sobre outros fatores, como por exemplo sobre a saúde do recém-nascido e da mãe. A taxa de natalidade nos estudos supracitados, é determinada por elementos como renda familiar, nível de escolaridade dos progenitores e estoque de capital humano na economia.

Dessa forma, dada a relevância do assunto, a presente pesquisa tem como objetivo verificar como é a relação entre o nível de educação e a taxa de natalidade nos municípios da Região Sul do Brasil, além de suas respectivas associaçóes espaciais. As variáveis do estudo foram levantadas da Federação das Indústrias do Estado do Rio de Janeiro (FIRJAN) e do Departamento de Informática do Sistema Único de Saúde (DATASUS). Neste caso, são o Índice FIRJAN de Desenvolvimento Municipal em Educação (IFDM - Educação) e o número de nascimentos por mil habitantes, respectivamente.

A Região Sul é uma das regióes que possui os melhores indicadores educacionais do Brasil, sendo por esse motivo o local em que este estudo foi aplicado, além de serem escassos os estudos que abordam essa temática nesta regiáo geográfica. Por outro lado, o ano de 2016 foi escolhido por ser o mais recente que possui dados compatíveis para o estudo em nível municipal.

Portanto, esta pesquisa contribui para o debate sobre taxa de natalidade pois, visto a importância da questão, objetiva-se evidenciar a existência de uma relação inversa entre as duas variáveis analisadas e que tal relação é espacialmente dependente, ou seja, transborda para os municípios vizinhos. Sendo assim, é esperado que municípios com elevado nível de educação seja cercado de municípios com baixa taxa de natalidade.

Além desta introdução, o artigo possui mais quatro seções. Na seçăo seguinte é apresentada uma revisão teórica e empírica sobre os estudos de natalidade. Em seguida, é exposta a metodologia aplicada para alcançar o objetivo. Posteriormente, os resultados e a discussão são apresentados e, por fim, as consideraçōes finais.

\section{A RELAÇÃO ENTRE EDUCAÇÃO E TAXA DE NATALIDADE}

A questáo da natalidade já era discutida desde o século XVIII, em que Malthus (1798) teorizava que quanto maior fosse a renda do indivíduo, mais cedo ele se casaria, 
tendo maior tempo para ter filhos, e por consequência, elevação da taxa de natalidade em sua regiáo. Contudo, Becker (1993) deixou evidente a simplicidade do modelo malthusiano por não se aplicar em economia moderna. Um dos fatores náo utilizados no modelo é a valoraçáo do tempo, o aumento deste fator faz com que se torne mais caro ter filhos, o que explica a redução de famílias numerosas. Por outro lado, outro fator não observado no modelo malthusiano era a importância do investimento privado em educação, em que os pais tendem a investir mais na educação de seus filhos em economias mais desenvolvidas, o que também gera menor interesse em ter um filho adicional, esboçando uma relação inversa entre nível de educação e taxa de natalidade.

Becker (1960) analisou os determinantes da fertilidade comparando as crianças com bens duráveis. Assim como os bens analisados, a fertilidade é determinada por outros fatores além da renda, como os custos de longo prazo com as crianças, em especial, a qualificação. Logo, não somente a demanda da família por um filho que determinaria o nascimento de uma criança, mas também a capacidade da família fornecer para a criança uma boa educação. Em um planejamento familiar adequado, os cônjuges possuem o entendimento da importância de uma boa educaçáo para os filhos, principalmente em economias com alto nível de capital humano.

Nesta questáo do fornecimento educacional, Marshall (1985) deixou claro que a obstruçáo do acesso dos jovens pobres a novas oportunidades atuava no sentido contrário ao surgimento de novos gênios, reduzindo as possibilidades de aumento da riqueza nacional. Por esse motivo, defendia que a educação deveria ser garantida pelo Estado e incentivada pelos pais. Consequentemente, o mérito deste processo causaria uma reduçáo na taxa de natalidade, visto que haveria êxito na educação de apenas uma criança, tornando desnecessário ter outro filho.

No estudo de Becker, Murphy e Tamura (1990) a fertilidade foi analisada de forma endógena, onde seu retorno de investimento foi comparado ao retorno do investimento em capital humano em dois cenários diferentes. No primeiro cenário, em economias com capital humano abundante, as taxas de retornos deste capital eram maiores do que as taxas de retornos da fertilidade. No segundo cenário, em economias com baixo nível de capital humano, as taxas de retornos do capital humano eram menores do que as taxas de retorno da fertilidade. Diante disso, verificou-se que economias mais desenvolvidas e com maiores níveis de educação possuem menores taxas de natalidade.

Outra questão a se levar em consideração é a educação feminina, embora os dados disponíveis a nível municipal não permitam uma análise desagregada por sexo neste estudo. A literatura científica considera que tanto nos países desenvolvidos, quanto nos países em desenvolvimento, as mulheres que possuem maiores níveis de escolaridade têm menos filhos do que aquelas com níveis mais baixos de educação. No entanto, as razóes para esse efeito são menos claras, pois existem vários aspectos da educaçáo que podem ser importantes para a natalidade (KIM, 2016).

A educação pode afetar a escolha das pessoas, em especial das mulheres, quanto a fertilidade e investimento infantil através da renda ou aprendizado (MICHAEL, 1973). A medida que as pessoas com maior nível educacional possuem renda mais elevada, elas acreditam que ter mais filhos se torna a opção mais cara e desejam ter menos filhos 
(KIM, 2016). Descobertas empíricas mostraram que o aumento da renda, principalmente da feminina via educação, está acompanhada pela redução no número de filhos. Como o aumento da educação é um determinante importante para o crescimento da renda, a educação feminina reduz indiretamente a fertilidade por meio de seus ganhos (MINCER, 1963; BECKER, 1965; BECKER; LEWIS, 1973; WILLIS, 1973).

A educação pode impactar de outras formas na taxa de natalidade, não se concentrando apenas no aumento da renda. Além desta, a literatura tem enfatizado o papel da educação no crescimento do estoque de conhecimento em saúde de um indivíduo (WILLIS, 1973; CURRIE; MORETTI, 2003; KIM, 2016). A educação permite melhor compreensão sobre saúde preventiva, fazendo com que as pessoas procurem por maiores informaçóes nesta questão. Dessa forma, a educação pode melhorar o estoque de conhecimento de uma mulher sobre métodos contraceptivos, comportamentos saudáveis durante a gravidez ou a utilizar de maneira mais frequente e correta os serviços e insumos de saúde (MCCRARY; ROYER, 2011).

Os indivíduos que possuem maiores níveis de educação apresentam maiores probabilidades de conhecer métodos contraceptivos modernos e adotar novos métodos de controle de natalidade, conforme Rosenzweig e Schultz (1989). De acordo com Kim (2016), evidências nos países desenvolvidos e em desenvolvimento têm mostrado que as diferenças na fertilidade entre mulheres com diferentes níveis de escolaridade estão fortemente correlacionadas com as diferenças no uso de contraceptivos.

Além do uso de métodos para o controle de natalidade, a educação permite aumentar a capacidade da mulher em adquirir e processar informaçóes sobre a saúde infantil, conforme as pesquisas de Thomas, Strauss e Henriques (1991) e de Mccrary e Royer (2011). As mulheres com maiores níveis de escolaridade têm maior conhecimento materno sobre, por exemplo, o pré-natal e nutrição infantil, o que pode influenciar de alguma forma sua fertilidade e terem crianças com melhores perspectivas de sobrevivência (KIM, 2016).

Outros estudos ajudam a apoiar este fato, como o de Currie e Moretti (2003) em que observaram que mulheres residentes dos Estados Unidos com maiores níveis educacionais são mais propensas a fazerem uso de pré-natais, não serem tabagistas e terem maiores probabilidades de serem casadas. Como resultados indiretos sobre o assunto, verificaram que um ano adicional de educação materna reduz a incidência de baixo peso ao nascer em cerca de $10 \%$ e de nascimentos prematuros em aproximadamente $6 \%$.

Ainda no que tange ao impacto da educação em variáveis demográficas, o estudo de Breierova e Duflo (2004) avaliou o programa de construção de escolas na Indonésia e mostrou que um ano adicional de escolaridade materna reduz a mortalidade infantil em cerca de um terço. Por fim, Chou et al. (2010) analisaram uma reforma do governo de Taiwan, onde em 1968 estenderam a educação obrigatória de seis para nove anos. O resultado foi que o aumento na educação da mãe em um ano levou a redução da mortalidade infantil entre $7 \%$ e $9 \%$.

Conforme verificado, três mecanismos podem influenciar a decisão de fertilidade: (1) a renda relativamente mais alta de pessoas mais escolarizadas; (2) os impactos positivos da educação na saúde, tanto para as mulheres quanto para seus filhos e (3) o impacto da 
educação no conhecimento sobre o uso de contraceptivos. Embora a relevância de cada mecanismo possa variar dependendo da região, do tempo e dos indivíduos analisados, são evidentes as presenças de associação espacial e de causalidade entre educação e taxa de a natalidade e, por isso, torna-se importante objeto de estudo.

\section{DADOS E METODOLOGIA}

\subsection{Dados}

Os dados utilizados nesta pesquisa são para o ano de 2016, este ano foi escolhido por conta da melhor adequação das observaçóes para as variáveis utilizadas na pesquisa, neste caso são 1187 municípios da Região Sul. Os dados foram retirados da Federação das Indústrias do Estado do Rio de Janeiro (FIRJAN) e do Departamento de Informática do Sistema Único de Saúde (DATASUS).

A primeira fonte fornece o IFDM - Educação, que é tratado como proxy de nível educacional neste estudo. Este índice é composto por atendimento à educação infantil, abandono no ensino fundamental, distorção idade-série no ensino fundamental, docentes com ensino superior completo que atuam no ensino fundamental, média de horas-aula e resultado do IDEB. Já a segunda fonte fornece o número de nascimento por município a cada mil habitantes (Nascimentos/MilHab).

\subsection{Estatística descritiva dos dados}

A Tabela 1 apresenta as médias em níveis agregados para cada estado, para a Região Sul e para o Brasil das variáveis IFDM - Educação e Nascimentos/MilHab. Observa-se que o estado da Região Sul com maior nível educacional é Santa Catarina, enquanto Rio Grande do Sul possui o menor nível. Na questão da fertilidade, o Paraná é o estado com maior taxa de natalidade, enquanto o Rio Grande do Sul é o menor. Isto reflete a importância de fazer a análise de forma desagregada em nível municipal para que a relação inversa entre as variáveis possa de fato ser evidenciada.

Tabela 1 - Média das variáveis de interesse

\begin{tabular}{l|c|c}
\hline & IFDM - Educaçáo & Nascimentos/MilHab \\
\hline PR & 0,813 & 13,276 \\
\hline SC & 0,837 & 12,606 \\
\hline RS & 0,805 & 10,373 \\
\hline Sul & 0,816 & 11,899 \\
\hline Brasil & 0,769 & 13,601 \\
\hline
\end{tabular}

Fonte: Elaborado pelos autores.

A Figura 1 apresenta a distribuição espacial das variáveis do estudo, é possível analisar que a maior concentração do IFDM - Educação acontece nos municípios ao Norte e ao 
Oeste do Paraná, ao Oeste e ao Leste de Santa Catarina, ao Norte e ao Noroeste do Rio Grande do Sul.

A qualidade da educação nestes municípios é maior do que nos outros munícipios da Região Sul, visto que o índice educacional é maior que a média do índice na região, 0,816. Em relação a taxa de natalidade, a maior concentração acontece no Centro e no Sul do Paraná, e no Centro de Santa Catarina, justamente onde existe menor qualidade educacional.

Figura 1 - Distribuição espacial das variáveis

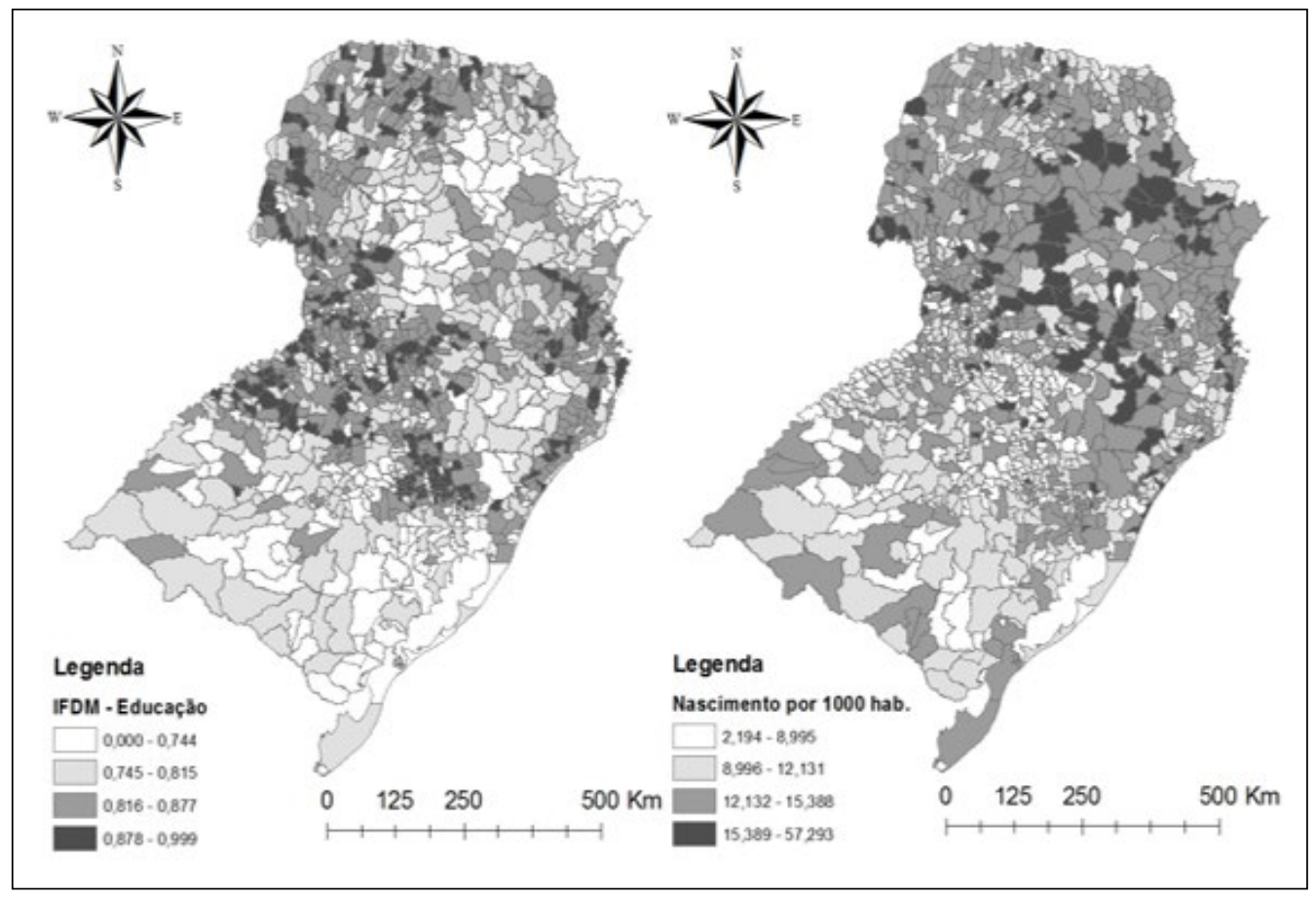

Fonte: Elaborado pelos autores.

Cabe ressaltar que nestas regiōes a renda per capita também é baixa, comparada aos demais municípios, o que pode influenciar o baixo nível educacional.

\subsection{Análise Exploratória de Dados Espaciais}

Para realizar a análise da distribuição espacial das variáveis para os municípios da Região Sul do Brasil, bem como analisar espacialmente a maneira que as duas variáveis estão relacionadas, foi utilizado o método da Análise Espacial de Dados Espaciais - AEDE. Método que possibilita visualizar, pela construção de gráficos e tabelas, a existência de algum tipo de padrão de associação espacial da variável em estudo, indicando assim a existência de regiōes com características semelhantes (clusters) e as com características diferentes (outliers). A aplicação dessa metodologia se justifica por se esperar que a distribuição dos municípios 
da Região Sul com elevada educação e taxa de natalidade não são distribuídos aleatoriamente na região (ANSELIN, 1988).

Para se aplicar o método AEDE, deve se estabelecer qual o critério que permitirá a avaliação da distribuição espacial das variáveis educação e natalidade. Tal critério é estabelecido por uma matriz de ponderação espacial, matriz $W$, e será ela que indicará o grau de conexão dos municípios. A escolha da matriz $W$ se dá pela sua capacidade em captar a relação espacial, que pode ser verificada por meio de adjacência, contiguidade ou distância geográfica. Por meio da relação de contiguidade, a matriz escolhida será binária, compostas por valores unitários, quando as regiōes apresentarem fronteira física e zeros quando não apresentarem. Nesse tipo de matriz, as mais utilizadas são torre e rainha, sendo que a primeira considera vizinhos as regióes que somente fazem fronteira no plano vertical e horizontal, e na segunda as que fazem na vertical, horizontal e diagonal. A crítica a escolha de tais matrizes é que elas não são balanceadas, uma vez que existe a possibilidade de uma região possuir vários vizinhos, enquanto outras, poucos vizinhos. (ALMEIDA, 2012).

Para se corrigir tal limitação exposta no parágrafo anterior, pode se empregar matrizes binárias que considerem critérios geográficos, sendo estas matrizes as que atribuem maior peso às regióes com maior proximidade, como no pressuposto da Lei de Tobler (quanto mais próximas duas regióes, maior será o efeito de uma variável da região $i$ na região $j$ ). No método AEDE, tal matriz é criada estabelecendo um número fixo $k$ de vizinhos mais próximos, possuindo o peso $W_{i j}(k)$.

Estabelecida a matriz que será utilizada, de acordo com Almeida (2012), a próxima etapa é verificar ou não a existência de algum padrão no comportamento das variáveis em estudo. Dentre os métodos de avaliação de tal comportamento, a partir da matriz espacial, um com extensa aplicação na literatura empírica é a estatística I de Moran, que pode ser global ou local. Esse coeficiente mostra qual o tipo de associação linear existente no espaço, e é obtido por meio da seguinte equação:

$$
\begin{aligned}
& I=\frac{n}{s_{0}} \frac{\sum_{i} \sum_{j} w_{i j} z_{i} z_{j}}{\sum_{i=1}^{n} z_{i}^{2}} \\
& \text { ou, matricialmente: }
\end{aligned}
$$

$$
I=\frac{n}{s_{0}} \frac{z^{\prime} W z}{z^{\prime} z}
$$

em que $n$ são as unidades geográficas. $z$ são valores da variável de interesse padronizada. $W z$ são valores médios da variável de interesse padronizada entre vizinhos, definida por uma matriz de ponderação espacial $W$. E $S_{0}$ representa a soma de todos os elementos das matrizes espaciais (ANSELIN; FLORAX; REY; 2013).

A interpretação do I de Moran é feita por meio de duas hipóteses, a nula e a alternativa. $\mathrm{Na}$ hipótese nula, se define a não dependência espacial, é obtida por meio do cálculo do I de Moran esperado, cujo a fórmula é $[1 / n-1)]$. A hipótese alternativa, dada pela equação 1 e 2, aponta a existência de algum tipo de padrão na distribuição espacial dos dados. Verificada a existência de dependência espacial, esta pode apresentar dois padróes, interpretado por meio do sinal do I de Moran. Caso seja positivo, o comportamento das 
unidades espaciais são os mesmos, ou seja, uma determinada região $i$ com alto valor para determinada variável, será vizinho de outras regióes $j$ que também possuem um alto valor. Caso o I de Moran seja negativo, o comportamento das unidades espaciais será diferente, de modo que uma determinada regiáo $i$ com alto valor para determinada variável, será vizinho de outras regióes $j$ que possuem um baixo valor (ALMEIDA, 2012).

O diagrama de dispersão do I de Moran, ilustrado na figura 2, é dividido em quatro quadrantes, a saber, Alto-Alto (AA), Baixo-Alto (BA), Baixo-Baixo (BB) e Alto-Baixo (AB). No quadrante $\mathrm{AA}$, estão as unidades geográficas que possuem elevado valor para à variável em análise e seus vizinhos também possuem essa característica. No quadrante BB, a análise é o oposto, ou seja, unidades geográficas que possuem baixo valor para variável com vizinhos que também possuem baixo valor. Os quadrantes $\mathrm{AB}$ e $\mathrm{BA}$ são aqueles onde a variável em estudo, em determinada região tem alto (baixo) valor, e é vizinha de unidades com baixo (alto) valor (FOTHERINGHAM, et al., 2002; ALMEIDA, 2012).

Figura 2 - Demonstração do gráfico de dispersão do I de Moran.

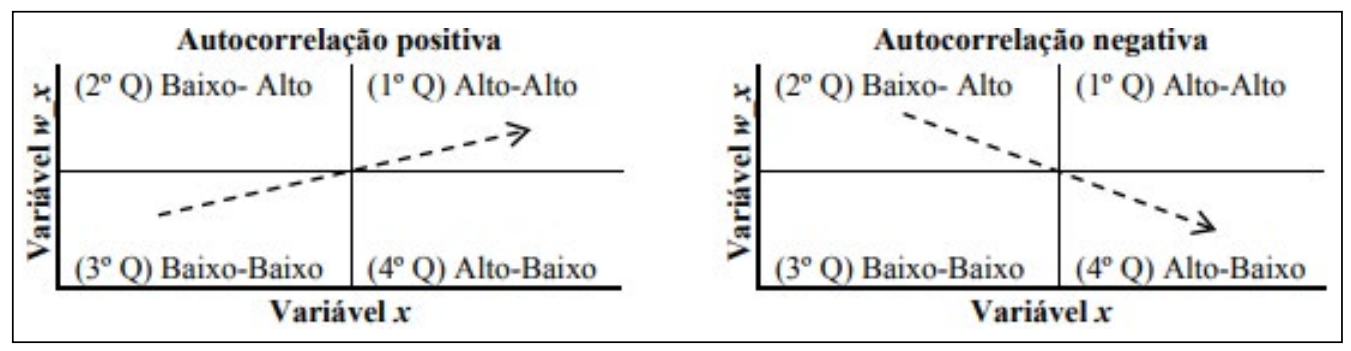

Fonte: Almeida (2012).

No estudo de dependência espacial, considerando apenas o aspecto global, tal análise tende a não considerar algum tipo de correlação espacial específico a alguma localidade, que mesmo existindo, não é captada. Para tratar essa potencial falha, pode-se empregar o I de Moran local, denominado por indicador Local Indicator of Spatial Association (LISA).

Nesse tipo de análise, só será considerada local se em cada observação existir algum clusters, sendo eles significantes e de valores similares em torno da observação e se a soma de todas LISA's, para todas as regióes, forem proporcionais ao indicador de autocorrelação espacial global (ANSELIN, 1995).

O coeficiente I de Moran local decompóe o indicador global de dependência espacial, e verifica a importância da observação entre as quatro categorias existente $(\mathrm{AA}, \mathrm{BB}, \mathrm{AB}$ e $\mathrm{BA}$ ). Dessa forma, cada regiáo isoladamente será representada em um dos quadrantes no diagrama de dispersão de Moran.

O I de Moran local, ao padronizar a variável em estudo $(y)$, observada na região $i, z_{i}$ , é obtido por meio da equação:

$$
I_{i}=z_{i} \sum_{j=1}^{j} w_{i j} z_{j}
$$

Nessa análise, para cada observação é calculado um $I_{i}$, além disso, obtém-se $n$ calculos da estatística $I_{i}$ e seus níveis de significância. Um meio de se apresentar o total dessas 
estatísticas é por meio de mapas de cluster LISA. Nesse tipo de representação dos dados, tem se a combinação das informaçōes do diagrama de dispersão de Moran com o mapa de significância das medidas de associação local. Além de ser permitido constatar as quatro categorias de correlação espacial que apresentaram significância estatística (ALMEIDA, 2012).

\section{RESULTADOS}

A Tabela 2 apresenta a escolha da matriz de ponderação espacial que foi utilizada para as análises seguintes. A matriz escolhida foi a de cinco vizinhos, K-5. Esta matriz apresentou o maior I de Moran calculado e significativo. Diante disso, os resultados serão em funçáo dos cinco vizinhos mais próximos do munícipio $i$.

Tabela 2 - Escolha da Matriz de Ponderação

\begin{tabular}{l|l|l}
\hline Matriz & I de Moran & P-Valor \\
\hline Torre & 0,2405 & 0,001 \\
\hline Rainha & 0,2407 & 0,001 \\
\hline K-5 & 0,2564 & 0,001 \\
\hline K-7 & 0,2419 & 0,001 \\
\hline K-10 & 0,2345 & 0,001 \\
\hline
\end{tabular}

Fonte: Elaborado pelos autores.

A Figura 3 apresenta o gráfico de dispersão do I de Moran e suas respectivas retas de correlação. Observa-se que na análise global univariada os municípios com alto nível de educação são vizinhos de municípios com alto nível de educação, e os municípios com alta taxa de natalidade são vizinhos de municípios com alta taxa de natalidade.

$\mathrm{Na}$ análise global bivariada entre as duas variáveis de interesse verifica-se que uma correlação negativa, que embora tenha baixa inclinação, também é estatisticamente significante. Portanto, pode-se afirmar que municípios que tenham alto nível educacional são vizinhos de municípios com baixa taxa de natalidade. O que corrobora os estudos de Willis (1973) e de Becker, Murphy e Tamura (1990) em que há um desestimulo à fertilidade quando existe alto nível de educação. 
Figura 3 - Gráfico de dispersão do I de Moran

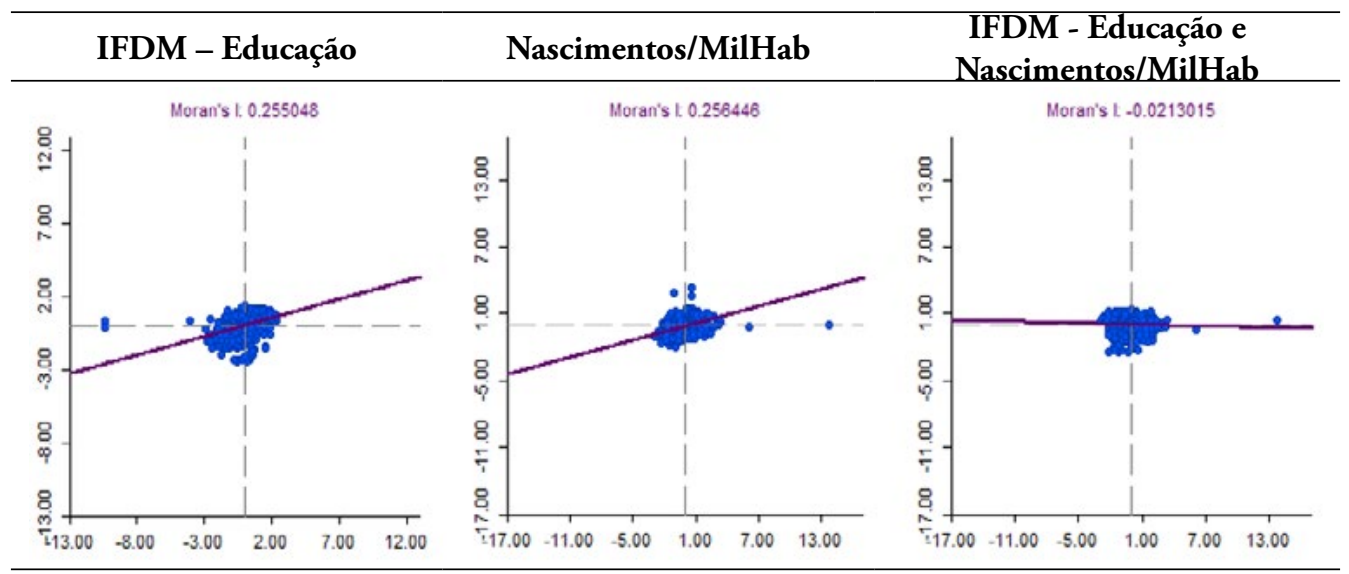

Fonte: Elaborado pelos autores.

Todas significativas estatisticamente a pelo menos 0,05.

Visto que o I de Moran global não possibilita uma análise de correlação espacial regional como aponta Almeida (2012), é necessário aplicar a análise local por meio da técnica LISA. Primeiramente é feita a análise local univariada e depois a análise local bivariada. A Figura 2 apresenta a correlação espacial univariada e local entre IFDM - Educação e Nascimentos/MilHab nos municípios da Região Sul.

Observa-se que na análise local univariada do IFDM - Educação, os clusters que predominam são "baixo-baixo", municípios que possuem baixo nível de educação são vizinhos de munícipios que também possuem baixo nível de educaçáo. Isto ocorre de maneira nítida no Sul e no Leste do Paraná, e no Sul do Rio Grande do Sul.

No tocante a taxa de natalidade, verifica-se que em parte do estado do Paraná existe clusters "alto-alto", portanto, municípios que se situam nesses clusters possuem alta taxa de natalidade e são vizinhos de municípios que também possuem alta taxa de natalidade. No Rio Grande do Sul a predominância é de clusters "baixo-baixo". Sendo assim, os municípios que se situam nesses clusters possuem baixa taxa de natalidade e são vizinhos de municípios que também possuem baixa taxa de natalidade. 
Figura 2 - Correlação espacial local e univariada do IFDM - Educação e Nascimentos/ MilHab.

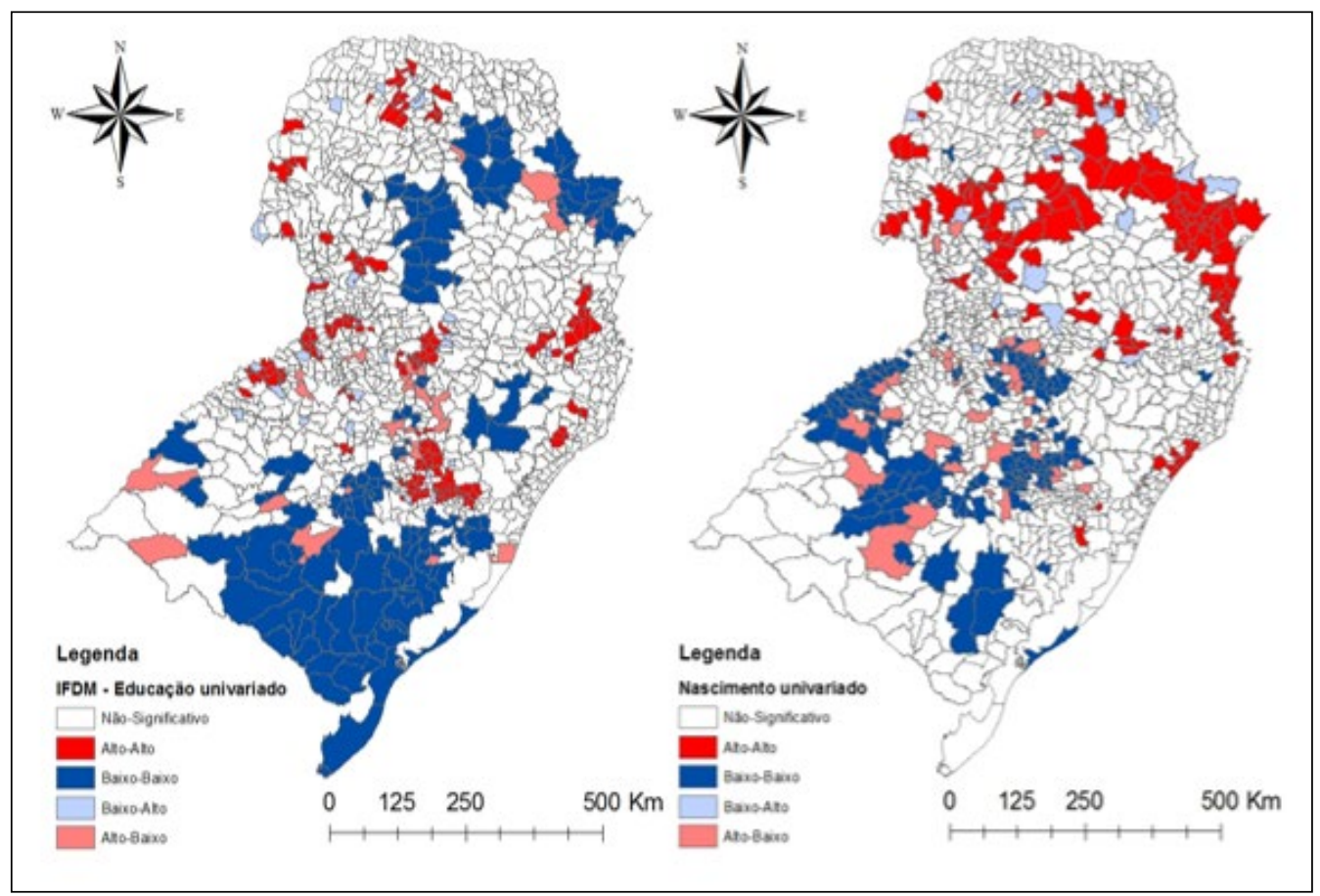

Fonte: Elaborado pelos autores.

$\mathrm{Na}$ análise bivariada apresentada pela Figura 3, observa-se que apesar de haver clusters "alto-alto" em alguns pontos do mapa, principalmente próximos às cidades de Marechal Candido Rondon, Maringá e Blumenau, existe a predominância dos clusters "baixo-baixo" no extremo Sul e dos clusters "alto-baixo" no Sul e no Leste do Paraná.

Portanto no Sul do Rio Grande do Sul, cidades com baixa taxa de natalidade são vizinhas de cidades com baixa qualidade na educação. Isto pode ser explicado porque nessa região do mapa concentra-se cidades que estão no primeiro quartil da distribuição geográfica das duas variáveis em questão (ver Figura 1). 
Figura 3 - Correlação espacial local e bivariada entre IFDM - Educação e Nascimentos/ MilHab.

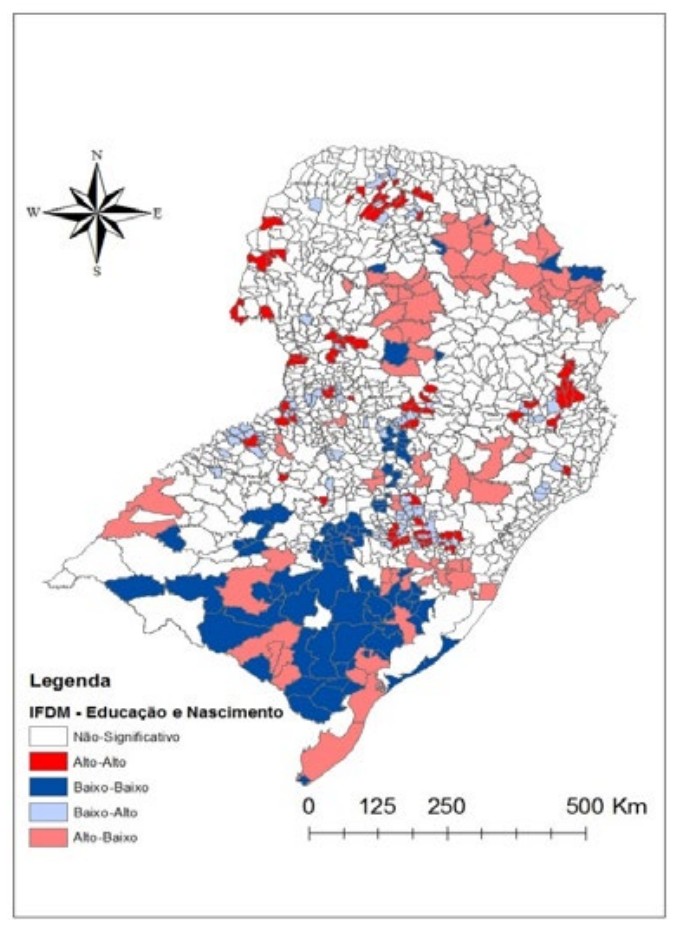

Fonte: Elaborado pelos autores.

No que se refere aos clusters "alto-baixo" no estado do Paraná, afirma-se que cidades do Sul e do Leste do estado que possuem alta taxa de natalidade, são vizinhas de cidades com baixa educação. Isto está relacionado ao fato de que essas regióes do estado do Paraná concentram cidades com qualidade da educação abaixo da média da Região Sul, 0,816. Além de taxa de natalidade acima da média da Região Sul, 11,90.

\section{CONSIDERAÇÓES FINAIS}

O objeto deste estudo foi a relação espacial entre o nível de educação e a taxa de natalidade nos municípios da Regiáo Sul, visto que são escassas as pesquisas neste âmbito para esta região, mesmo possuindo bons níveis de educação formal comparada à outras regiốes do país. A relevância deste estudo está pautada no fato de que ele contribui para os cenários econômicos e geográficos, uma vez que as decisões do agente humano são dinâmicas e podem influenciar na sua alocação espacial, assim como em sua reprodução.

A hipótese levantada no estudo, de haver uma associaçáo inversa entre nível de educaçáo e taxa de natalidade na Regiáo Sul foi confirmada em uma análise global. Entretanto, na análise local é possível verificar certas características especificas em alguns pontos dentro da Regiáo Sul, como são os casos do Sul e do Leste paranaense, e do Sul do Rio Grande do Sul. 
Embora esse estudo não tenha a pretensão de esgotar as discussóes do tema que é tâo amplo, especialmente no seio da Economia e da Geografia, ele procura contribuir com os formuladores de políticas públicas, no sentido de que os mesmos tenham ao seu alcance maiores rudimentos das variáveis aqui analisadas. Para que promovam nos âmbitos de sua atuação, políticas públicas que, caso objetivem ter maior controle sobre a natalidade em longo prazo, principalmente em municípios mais pobres e com sistemas de saúde sobrecarregados, devem levar em consideração como um dos principais fatores de decisão a elevação do nível da educação formal.

\section{REFERÊNCIAS}

ALMEIDA, E. Econometria Espacial Aplicada. Alínea: Campinas-SP, 2012.

ANSELIN, L. Local indicators of spatial association-LISA. Geographical analysis, p. 93-115, 1995. Disponível em: https://onlinelibrary.wiley.com/doi/ abs/10.1111/j.1538-4632.1995.tb00338.x. Acesso em: 10/11/2019.

ANSELIN, L. Spatial econometrics: methods and models. Springer Science \& Business Media, 2013.

ANSELIN, L.; FLORAX, R.; REY, S. J. Advances in spatial econometrics: methodology, tools and applications. Springer Science \& Business Media, 2013.

BECKER, G. S. An economic analysis of fertility. In: Demographic and economic change in developed countries. Columbia University Press, p. 209-240, 1960. Disponível em: https://www.nber.org/chapters/c2387.pdf. Acesso em: 17/11/2019.

BECKER, G. S. A Theory of the Allocation of Time. Economic Journal, v. 75, n. 299, p. 493-517, 1965.

BECKER, G. S.; LEWIS, H. G. On the Interaction between the Quantity and Quality of Children. Journal of Political Economy, v. 81, n. 2, p. 279-88, 1973.

BECKER, G. S. Nobel lecture: The economic way of looking at behavior. Journal of political economy, p. 385-409, 1993. Disponível em: https://www.journals.uchicago. edu/doi/abs/10.1086/261880. Acesso em: 26/11/2019.

BECKER, G. S.; MURPHY, K. M.; TAMURA, R. Human Capital, Fertility, and Economic Growth. Chicago-Population Research Center, 1990. Disponível em: https:// ideas.repec.org/p/fth/chiprc/90-5a.html. Acesso em: 23/11/2019.

BREIEROVA, L.; DUFLO, E. The Impact of Education on Fertility and Child Mortality: Do Fathers Really Matter Less Than Mothers? National Bureau of Economic Research, 2004. 
CHOU, S. Y.; LIU, J. T.; GROSSMAN, M.; JOYCE., T. J. Parental education and child health: Evidence from a natural experiment in Taiwan. American Economic Journal: Applied Economics, v. 2, n. 1, p. 33-61, 2010.

CURRIE, J.; MORETTI, E. Mother's education and the intergenerational transmission of human capital: evidence from college openings. The Quartely Journal of Economics, v. 118, n. 4, p. 1495-1532, 2003.

FOTHERINGHAM, A. S.; BRUNSDON, C.; CHARLTON, M. Geographically weighted regression: the analysis of spatially varying relationships. John Wiley \& Sons, 2003.

KIM, J. Female education and its impact on fertility. IZA World of Labor, 2016.

MALTHUS, T. R. An essay on the principle of population as it affects the future improvement of society, with remarks on the speculations of Mr Godwin, $M$. Condorcet, and other writers. London: J. Johnson, 1798.

MARSHALL, A. Princípios de economia: tratado introdutório, 1985.

MCCRARY, J.; ROYER, H. The Effect of Female Education on Fertility and Infant Health: Evidence from School Entry Policies Using Exact Date of Birth. American Economic Review, v. 101, n. 1, p. 158-195, 2011.

MICHAEL, R. T. Education and the Derived Demand for Children. Journal of Political Economy, v. 81, n. 2, p. 128-64, 1973.

MINCER, J. Market prices, opportunity costs, and income effects. Measurement in economics, p. 67-82, 1963.

ROSENZWEIG, M. R.; SCHULTZ, T. P. Schooling, Information and Nonmarket Productivity: Contraceptive Use and Its Effectiveness. International Economic Review, v. 30, n. 2, p. 457-77, 1989.

THOMAS, D.; STRAUSS, J.; HENRIQUES, M. H. How Does Mother's Education Affect Child Height? Journal of Human Resources, v. 26, n. 2, p. 183-211, 1991.

WILLIS, R. J. A New Approach to the Economic Theory of Fertility Behavior. Journal of Political Economy, v. 81, n. 2, p. 14-64, 1973. 